\section{A CASE OF POISONING BY CAMPHORATED} OIL.

BY FREDERIC BARKER, M.B., B.S.LOND,

L.R.C.P., M.R.C.S., NORTHWICH.

Camphorated oil (Linimentum camphorae, B.P.) is a common domestic remedy, often supplied to the public in bottles which do not indicate by shape or label the poisonous nature of their contents. Camphor poisoning, nevertheless, does not appear to be of frequent occurrence, and the following notes may be of interest; but it is my desire to advocate the adoption of suitable precautions by those who sell the oil, for had the bottle in this case shown the need for care in handling, a child's life would not have been lost.

I was called on February 3rd to see a female child, aged 1 year and 4 months. She had been put to bed earlier in the eveving, and had been given a bottle of camphorated oil to play with. At 7 p.m. a brother shouted out that the baby was "drinking castor-oil":; the father found the child with its mouth full of the oil. He promptly turned her over and she "vomited on the floor." She remained well until 8 p.m., when she vomited again. The eyes gradually became staring, she moaned, and at 8.20 p.m. convulsions began. She was then given some salt and water; and vomiting occurred.

When I saw her at 9 p.m. there were very marked convulsions, with irregnlar breathing, flushed face and profuse perspiration the pulse was rapid, the pupils were moderate and equal in size areath did not smell of camphor, and the mouth was not sore. The baby was put in a hot bath, with cold cloths to the bead, while I prepared a glass funnel, some tubing and a soft rubber catheter as a stomach tube. The stomach was washed out with warm water until quite clear of oil and débris, the washings being placed in four successive bowls. The first two contained food remains in small quantity, with a considerable amount of food remains in small quantity, with a considerable amount of oil floating on the top, smelling distinctly of camphor.
last two showed the merest film of oil and did not smell.

At 10.40 p.m. the pulse was about 200 , temperature in rectum
. the (taken twice) 106.4'. Remedies seemed to have little or no effect. Chloral hydrate (3 grains) by the rectum, small doses of potassium bromide by stomach tube and by rectum. hot milk and water similarly given, and brandy were tried. About midnight I gave chloroform very cautiously, and with even the most sparing dosage the fits were to some extent controlled most they became periodic, and in the intervals the breathing was so markedly stertorous, and the face more cyanosed than before, that I seased giving the anaesthetic. While giving chloroform the fits were heralded by a flickering of the eyelids the spasms spreading to the abdominal and back muscles and then to the extremities. There was usually at first conjugate deviation of the eyes and drawing of the mouth to the left, followed by the turning of the eyes and mouth to the right. On ceasing the chloroform the convalsions became more continuous but the chloroform the convulsions became more continuous but
were not so violent, the breathing was decidedly better, and the face a more natural colour. Later there was a slight return of conjunctival reflex. About 5 a.m. collapse set in, and the baby died about 6.30. I was not present at the end, but the father thought the child was "a bit conscious."

The bottle of oil, which was sold by a co-operative society, contained at the time of the misadventure approximately ten drachms. There remained in the bottle seventy minims, and the total content of the bottle was slightly over thirteen drachms. Over one ounce has therefore to be accounted for, and allowing for what was ejected in the turning over of the baby and some that was spilt on the bedclothes, it is probable that at least half an ounce was swallowed. The child was said to have vomited three times before I saw her, and there was vet a fair quantity (I should say over a teaspoonful) in the stomach washings. The composition of camphorated oil is camphor, in flowers, 1 ; olive oil, 4; so that about one drachm of solid camphor in solution was probably taken.

The gradual onset is worthy of note; it was not until an hour had passed that any symptoms developed, and there is no doubt that if the stomach had been washed out before absorption had taken place to any great extent the baby would have been saved. Apart from the spasmodic action of the respiratory muscles, which joined in the general convalsions, the poison seemed to me to have affected especially the respiratory centre.

The bottle is of flat shape, in plain glass, and is labelled merely "Camphorated Oil," with the name and address of the wholesale firm.

\section{THE TREATMENT OF VARICOSE VEINS OF THE LOWER LIMB BY AVULSION.}

By H. D. O'SUllivan, B.A., M.B., B.C.Cantab, BURTON-ON-TRENT.

I DESIRI to describe a method of extirpating varicose veins of the lower extremity which is successful, and which to me at least is entirely new. The concise description of an actual case will make the method clear, and also enable me to call attention to some interesting points in minor surgery.

The patient was a man, aged about 30 , who wished safely to engage in a game of football or hockey. The right lower limb exhibited three large, moderately tortuous veins. One extended from the front of the ankle to the inner side of the knee. Another was definitely varicose for two-thirds of the distance between ankle and knee. The third extended over half that distance. Above the ankle the three communicated by large and tortuous cross channels. The common origin appeared as a single vein in front of the ankle.

\section{Operation.}

On the evening prior to the operation the limb was shaved and then washed for half an hour with white soap and water. The veins to be removed were then painted with tincture of iodine (B.P.), the site of the proposed incisions being marked by for the night in a household towel fresh from the wash. On the morning of the operation, the washing and marking of the limb were exactly repeated. An elastic bandage was applied to the limb, and a tourniquet above the knee. A local to the limb, and a tourniquet above the knee, A local $1 \mathrm{oz}$.) sterilized by exposure to a temperature of $65^{\circ} \mathrm{C}$. for one hour on three successive days. I have, however, strong suspicion that distilled water widely inflltrated is as good as any solntion.

The longest vein was first dealt with, and the others in exactly similar fashion. It was exposed, immediately belotw the knee, by a flail-shaped incision, the lesser limb crossing the vein from above downwards. The upper end was tied and allowed to retract after division of the vein. The common origin of the veins at the ankle was next dealt with in similar fashion, the lower end being tied.

The longest vein was then exposed and divided by two incisions equidistant from each other and from the ankle and knee respectively. The cross channels were also exposed at either end and divided. This is essential.

A silver probe, with an eye threaded with stout silk, was then passed into the vein from below upwards. The probe was then passed along the lumen of the vein till it emerged at the incision next above. The silk was then firmly tied to the free end of the vein. A firm steady jerk then removed the whole functional vein between the two said incisions. The portions of veins between all the other incisions were then dealt with in similar fashion. The total length of the combined incisions was about 7 in. After suture the incisions were painted wint
tincture of iodine, and covered with pads of plain boiled lint.

On relaxing the tourniquet profuse haemorrbage occurred from each wound, which was easily controlled by the simple pressure of the usual bandage. The blood became diffased throughout the pads and bandage, and the whole quickly set to form a firm and comfortable splint.

The bandage and pads were removed on the fourth day. The limb was perfectly free from extravasation and soiling. The wounds were painted with tincture of iodine, and wool and a bandage applied. The sutures were removed on the tenth day. On the fourteenth day the patient got up and walked.

The patient, who comes of a family of nervous temperament, felt nothing of the operation itself, but during the last quarter of an hour complained a little of the tourniquet.

The novelty of the operation lies in the complete extirpation of the affected veins through small incisions. In this case some 25 in. of vein were removed through incisions of the total length of 7 in.

THE foundation stone of new barracks for British troops has recently been laid in Cairo; the site is between the Abbassia and the Poligon barracks.' When completed the new barracks will cover some forty acres, and be available for cavalry and infantry and for married quarters, and will be healthier and more comfortable than those now occupied, which are old and more or less out of repair. The new barracks, which will be built of artificial stone, will consist of detached blocks, each sufficient for a squadron of cavalry or company of infantry, with dining hall, baths, cold and hot water, and other conveniences. Spacious verandahs will be provided, and the artificial lighting will probably be electric. It is stated that the infantry barracks will be ready for occupation in about two years, when the Kasr.el-Nil troops will be moved to them. 